\title{
ON THE BRIANÇON-SKODA THEOREM ON A SINGULAR VARIETY
}

\author{
MATS ANDERSSON \& HÅKAN SAMUELSSON \& JACOB SZNAJDMAN
}

\begin{abstract}
Let $Z$ be a germ of a reduced analytic space of pure dimension. We provide an analytic proof of the uniform BriançonSkoda theorem for the local ring $\mathcal{O}_{Z}$; a result which was previously proved by Huneke by algebraic methods. For ideals with few generators we also get much sharper results.
\end{abstract}

\section{IntRoduction}

Let $\mathfrak{a}=(a)=\left(a_{1}, \ldots, a_{m}\right)$ be an ideal in the local ring $\mathcal{O}=\mathcal{O}_{0}$ of holomorphic functions at $0 \in \mathbb{C}^{d}$ and let $|\mathfrak{a}|=\sum_{j}\left|a_{j}\right|$. Up to constants, this function is independent of the choice of generators of $\mathfrak{a}$. In [13], Briançon and Skoda proved:

If $\phi \in \mathcal{O}$ and

$$
|\phi| \leq C|\mathfrak{a}|^{\min (m, d)+\ell-1}, \quad \ell=1,2,3, \ldots,
$$

then $\phi \in \mathfrak{a}^{\ell}$.

If $m \leq d$, then the statement follows directly from Skoda's $L^{2}$ estimate in [26]; if $m>d$ one uses that there is an ideal $\mathfrak{b} \subset \mathfrak{a}$ such that $|\mathfrak{a}| \sim|\mathfrak{b}|$, a so-called reduction of $\mathfrak{a}$, with $n$ generators.

If $\mathfrak{b}$ is any ideal in $\mathcal{O}$ then $|\phi| \leq C|\mathfrak{b}|$ if (and in fact only if) $\phi$ is in theintegral closure $\overline{\mathfrak{b}}$. Therefore, the statement implies (is equivalent to) the inclusion

$$
\overline{\mathfrak{a}^{\min (m, d)+\ell-1}} \subset \mathfrak{a}^{\ell} .
$$

This is a notable example of a purely algebraic theorem that was first proved by transcendental methods. It took several years before algebraic proofs appeared, [22] and [21]. In [11] there is a proof by integral formulas and residue theory.

Assume now that $Z$ is a germ of an analytic space of pure dimension $d$ and let $\mathcal{O}_{Z}$ be its structure ring of germs of (strongly) holomorphic functions. It is non-regular if (and only if) $Z$ is non-regular. It is easy to see that the usual Briançon-Skoda theorem cannot hold in general in the non-regular case, not even for $m=1$, see Example 1

Date: October 28, 2018.

The authors would like to thank the Institut Mittag-Leffler (Djursholm, Sweden) where parts of this work were carried out. The first author was partially supported by the Swedish Research Council. 
below. However, Huneke proved in [17] that there is a number $\mu$ only depending on $Z$ such that for any ideal $\mathfrak{a} \subset \mathcal{O}_{Z}$, and integer $\ell \geq 1$,

$$
\overline{\mathfrak{a}^{\mu+\ell-1}} \subset \mathfrak{a}^{\ell} .
$$

Huneke's proof is completely algebraic (and holds for some more general rings as well), so it is natural to look for an analytic proof. In this paper we give a proof by means of residue calculus, and the membership can be realized by an integral formula on $Z$. A problem of general interest, see, e.g., p. 657 in [18] and Remark 4.14 in [17], is to estimate the Briançon-Skoda number, $\mu$, in Huneke's theorem in terms of invariants of the ring. Our proof relates $\mu$ to the complexity of a free resolution of $\mathcal{O}_{Z}$. We have also a sharper statement in case $\mathfrak{a}$ has "few" generators, and the zero set, $Z^{\mathfrak{a}}$, of the ideal does not overlap the singular set of $Z$ "too much". To formulate this we first have to introduce certain (germs of) subvarieties, $Z^{r}$, associated with $Z$ :

To begin with we choose an embedding of $Z$ and consider it as a subvariety at, say, the origin of $\mathbb{C}^{n}$ for some $n$. If $\mathcal{I}$ is the corresponding radical ideal in $\mathcal{O}=\mathcal{O}_{\mathbb{C}^{n}, 0}$, then $\mathcal{O}_{Z}=\mathcal{O} / \mathcal{I}$. Let

$$
0 \rightarrow \mathcal{O}\left(E_{N}\right) \stackrel{f_{N}}{\longrightarrow} \ldots \stackrel{f_{3}}{\longrightarrow} \mathcal{O}\left(E_{2}\right) \stackrel{f_{2}}{\longrightarrow} \mathcal{O}\left(E_{1}\right) \stackrel{f_{1}}{\longrightarrow} \mathcal{O}\left(E_{0}\right)
$$

be a free resolution of $\mathcal{O} / \mathcal{I}$. Here $E_{k}$ are trivial vector bundles and $E_{0}$ is a trivial line bundle. Thus $f_{k}$ are just holomorphic matrices in a neighborhood of 0 . We let $Z_{k}$ be the set of points $x$ such that $f_{k}(x)$ does not have optimal rank. These varieties are, see, [15] Ch. 20, independent of the choice of resolution, and we have the inclusions

$$
\cdots \subset Z_{p+2} \subset Z_{p+1} \subset Z_{\text {sing }} \subset Z_{p}=\cdots=Z_{1}=Z,
$$

where $p=n-d$. Now let

$$
Z^{0}=Z_{\text {sing }}, \quad Z^{r}=Z_{p+r}, r>0 .
$$

Since any two minimal embeddings are equivalent, and any embedding factors in a simple way over a minimal embedding, one can verify that these subsets $Z^{r}$ are intrinsic subvarieties of the analytic space $Z$, that reflect the degree of complexity of $Z$. To begin with, since $Z$ has pure dimension (Corollary 20.14 in [15]),

$$
\operatorname{codim} Z^{r} \geq r+1, \quad r>0 \text {. }
$$

Moreover, $Z^{r}=\emptyset$ for $r>d-\nu$ if and only if the depth of the ring $\mathcal{O}_{Z}$ is at least $\nu$. In particular, $Z^{r}=\emptyset$ for $r>0$ if and only if $Z$ (i.e., $\mathcal{O}_{Z}$ ) is Cohen-Macaulay.

Theorem 1.1. Let $Z$ be a germ of an analytic space of pure dimension. (i) There is a natural number $\mu$, only depending on $Z$, such that for any ideal $\mathfrak{a}=\left(a_{1}, \ldots, a_{m}\right)$ in $\mathcal{O}_{Z}$ and $\phi \in \mathcal{O}_{Z}$,

$$
|\phi| \leq C|\mathfrak{a}|^{\mu+\ell-1}
$$

implies that $\phi \in \mathfrak{a}^{\ell}$. 
(ii) If for a given ideal $\mathfrak{a}=\left(a_{1}, \ldots, a_{m}\right)$

$$
\operatorname{codim}\left(Z^{r} \cap Z^{\mathfrak{a}}\right) \geq m+1+r, \quad r \geq 0,
$$

then for any $\phi \in \mathcal{O}_{Z}$,

$$
|\phi| \leq C|\mathfrak{a}|^{m+\ell-1}
$$

implies that $\phi \in \mathfrak{a}^{\ell}$.

Huneke's theorem (1.3) follows immediately from part (i) of Theorem 1.1, since even in the non-regular case $\phi \in \overline{(b)}$ immediately implies that $|\phi| \leq C|b|$. The less obvious implication $|\phi| \leq C|b| \Rightarrow \phi \in \overline{(b)}$ also holds, see, e.g., [20, and so Theorem 1.1 (i) is in fact equivalent to Huneke's theorem.

Example 1. If $Z$ is the zero set of $z^{p}-w^{2}$ in $\mathbb{C}^{2}$, where $p>2$ is a prime, then $|w| \leq|z|^{[p / 2]}$ on $Z$, but $w$ is not in $(z)$. However, if $|\phi| \leq C|z|^{(p+1) / 2}$, then $\phi \in(z)$, i.e., $\phi / z$ is strongly holomorphic on $Z$.

Remark 1. The important point in Huneke's theorem is the uniformity in $\mathfrak{a}$ and $\ell$. Notice that (1.3) implies the slightly weaker statement

$$
\overline{\mathfrak{a}}^{\mu+\ell-1} \subset \mathfrak{a}^{\ell} .
$$

It is quite easy to prove such an inclusion for fixed $\mathfrak{a}$ and $\ell$. In fact, assume that $Z$ is a germ of a subvariety in $\mathbb{C}^{n}$ and choose a tuple $f$ such that $Z=\{f=0\}$. Let $A=\left(A_{1}, \ldots, A_{m}\right)$ and $\Phi$ denote fixed representatives in $\mathcal{O}_{\mathbb{C}^{n}}$ of $\mathfrak{a}=\left(a_{1}, \ldots, a_{m}\right)$ and $\phi \in \overline{\mathfrak{a}}$. Then

$$
|\Phi(z)| \leq C d\left(z, Z^{\mathfrak{a}} \cap Z\right) \leq C^{\prime}(|A|+|f|)^{1 / M}
$$

for some $M$ by Lojasiewicz' inequality, and hence $\Phi^{M n}$ is in the ideal $(A)+(f)$ by the usual Briançon-Skoda theorem in the ambient space. Thus $\phi^{M n} \in \mathfrak{a}$ and therefore $\phi^{M n \ell} \in \mathfrak{a}^{\ell}$. Thus $\overline{\mathfrak{a}}^{M n \ell} \subset \mathfrak{a}^{\ell}$.

From Theorem 1.1 (ii) we get:

\section{Corollary 1.2. If}

$$
\operatorname{codim} Z^{r} \geq m+1+r, \quad r \geq 0,
$$

then (1.8) implies that $\phi \in \mathfrak{a}^{\ell}$ for any $\mathfrak{a}$ with $m$ generators.

Assume that (1.10) holds for $m=1$. The conclusion for $\ell=1$ then is that each weakly holomorphic function is indeed holomorphic, i.e., $Z$ (or equivalently $\mathcal{O}_{Z}$ ) is normal. In fact, if $\phi$ is weakly holomorphic, i.e., holomorphic on $Z_{\text {reg }}$ and locally bounded, then it is meromorphic, so $\phi=g / h$ for some $g, h \in \mathcal{O}_{Z}$. The boundedness means that $|g| \leq C|h|$ and by the corollary thus $\phi$ is in $\mathcal{O}_{Z}$. One can check that (1.10) with $m=1$ is equivalent to Serre's condition for normality of the local ring $\mathcal{O}_{Z}$ and therefore both necessary and sufficient. 
The basic tool in our proof is the residue calculus developed in [1, [7, and [8, and we recall the necessary material in Section 2. Given an ideal sheaf $\mathcal{J}$ one can associate a current $R$ such that a holomorphic function $\phi$ is in $\mathcal{J}$ as soon as $\phi R=0$. We use such a current $R^{\mathfrak{a}, \ell}$ associated with the ideal $\mathfrak{a}^{\ell}$. For $\ell=1$ it is the current of Bochner-Martinelli type from [1], whereas for $\ell>1$ we use a variant from [4]. Since we are to prove the membership on $Z$ rather than on some ambient space, thinking of $Z$ as embedded in some $\mathbb{C}^{n}$, we will also use a current $R^{Z}$ associated to the radical ideal $I$ of the embedding. For the analysis of this current we rely on results from [6], described in Section 3. It turns out that one can form the "product" $R^{\mathfrak{a}, \ell} \wedge R^{Z}$ such that $\phi R^{\mathfrak{a}, \ell} \wedge R^{Z}$ only depends on the values of $\phi$ on $Z$; moreover, if the hypotheses in Theorem 1.1 are fulfilled then it vanishes (Proposition 4.1), which in turn implies that $\phi$ belongs to the ideal $\mathfrak{a}$ modulo $I$. In the last section we present an integral formula that provides an explicit representation of the membership.

\section{Currents obtained from locally fReE COMPleXes}

Let

$$
0 \rightarrow E_{N} \stackrel{f_{N}}{\longrightarrow} E_{N-1} \stackrel{f_{N-1}}{\longrightarrow} \cdots \stackrel{f_{2}}{\longrightarrow} E_{1} \stackrel{f_{1}}{\longrightarrow} E_{0} \rightarrow 0
$$

be a generically exact holomorphic complex of Hermitian vector bundles over a complex manifold $X$, say a neighborhood of the origin in $\mathbb{C}^{n}$. We assume that $E_{0}$ is a trivial line bundle so that $\mathcal{O}\left(E_{0}\right)=\mathcal{O}$. There is an associated complex, like (1.4), of (locally) free sheaves of $\mathcal{O}$-modules, and we let $\mathcal{J}=f_{1} \mathcal{O}\left(E_{1}\right) \subset \mathcal{O}$ be the ideal sheaf generated by (the entries in) $f_{1}$. Let $Z$ be the analytic set where (2.1) is not pointwise exact. In $X \backslash Z$ we let $\sigma_{k}$ be the section of $\operatorname{Hom}\left(E_{k-1}, E_{k}\right)$ that vanishes on the orthogonal complement of the pointwise image of $f_{k}$ and is the minimal left inverse of $f_{k}$ on the image of $f_{k}$. If $E=\oplus E_{k}$, $f=\oplus f_{k}$, and $\sigma=\oplus \sigma_{k}$, then $\sigma f+f \sigma=I$, where $I$ is the identity on $E$. Since $E_{0}$ is trivial we identify $\operatorname{Hom}\left(E_{0}, E\right)$ with $E$. Following [7], in $X \backslash Z$ we define the form-valued sections

$$
u=\sum_{k=1}^{N} u_{k}, \quad u_{k}=\left(\bar{\partial} \sigma_{k}\right) \cdots\left(\bar{\partial} \sigma_{2}\right) \sigma_{1},
$$

of $E$. If $\nabla_{f}=f-\bar{\partial}$ we have that $\nabla_{f} u=1$. It turns out that $u$ has a current extension $U$ to $X$ as a principal value current: If $F$ is a tuple of holomorphic functions such that $F=0$ on $Z$, then $|F|^{2 \lambda} u$ has a current-valued analytic continuation to $\operatorname{Re} \lambda>-\epsilon$ and $U$ is the value at $\lambda=0$. Alternatively one can take a smooth approximand of the characteristic function $\chi$ for $[1, \infty)$, and let $\chi_{\delta}=\chi\left(|F|^{2} / \delta^{2}\right)$. Then $U$ is the weak limit of $\chi_{\delta} u$ when $\delta \rightarrow 0$ (see, e.g., the proofs of Theorems 16 and 21 in [24]). In this paper the latter definition will be more 
convenient. Clearly $\nabla_{f}$ also applies to currents, and

$$
\nabla_{f} U=1-R
$$

where $R$ is a residue current with support on $Z$; more precisely $R=$ $\lim _{\delta \rightarrow 0} R^{\delta}$, where

$$
R^{\delta}=R_{0}^{\delta}+R_{1}^{\delta}+R_{2}^{\delta}+\cdots=\left(1-\chi_{\delta}\right)+\bar{\partial} \chi_{\delta} \wedge u_{1}+\bar{\partial} \chi_{\delta} \wedge u_{2}+\cdots ;
$$

notice that $R_{k}^{\delta}$ is an $E_{k}$-valued $(0, k)$-current.

A basic observation is that the annihilator sheaf, ann $R$, of $R$ is contained in the sheaf $\mathcal{J}$, i.e.,

$$
\text { ann } R \subset \mathcal{J} \text {. }
$$

In fact, if $\phi \in \mathcal{O}$ and $\phi R=0$, then by (2.3),$\nabla_{f}(\phi U)=\phi-\phi R=\phi$. By solving a sequence of $\bar{\partial}$-equations, which is always possible locally at least, we get a holomorphic solution $\psi \in \mathcal{O}\left(E_{1}\right)$ to $f_{1} \psi=\phi$, which means that $\phi$ is in the ideal $\mathcal{J}$. One can also prove (2.4) by an integral formula that gives an explicit realization of the membership of $\phi$ in $\mathcal{J}$, see Section 5 .

In general the converse inclusion is not true. However, if the associated sheaf complex is exact, i.e., a resolution of $\mathcal{O} / \mathcal{J}$, then indeed ann $R=\mathcal{J}$ (Theorem 1.1 in [7]).

Example 2. Let $a_{1}, \ldots, a_{m}$ be holomorphic functions in $X$. Choose a nonsense basis $\left\{e_{1}, \ldots, e_{m}\right\}$ and consider $E_{1}=\operatorname{sp}\left\{e_{j}\right\}$ as a trivial vector bundle of rank $m$, let $e_{j}^{*}$ be the dual basis, and consider $a=$ $a_{1} e_{1}^{*}+\cdots+a_{m} e_{m}^{*}$ as a section of the dual bundle $E_{1}^{*}$. If $E_{k}=\Lambda^{k} E_{1}$ we then get a complex (2.1), the Koszul complex, with the mappings $f_{k}$ as interior multiplication $\delta_{a}$ with $a$. Following the recipe above (with the trivial metric on the $E_{k}$ ) we get, cf., 7] Example 1, the corresponding form

$$
u^{a}=\sum_{k=1}^{m} \frac{\left(\sum_{j=1}^{m} \bar{a}_{j} e_{j}\right) \wedge\left(\sum_{j=1}^{m} \bar{\partial} \bar{a}_{j} \wedge e_{j}\right)^{k-1}}{|a|^{2 k}}
$$

outside $\{a=0\}$ and the associated residue current $R^{a}=\lim _{\delta \rightarrow 0} R^{a, \delta}$ where $R^{a, \delta}=\left(1-\chi_{\delta}\right)+\bar{\partial} \chi_{\delta} \wedge u^{a}$ and $\chi_{\delta}=\chi\left(|a|^{2} / \delta^{2}\right)$. This current of so-called Bochner-Martinelli type was introduced already in [23], and its relation to the Koszul complex and division problems was noticed in [1]. Now (2.4) means that

$$
\text { ann } R^{a} \subset(a) .
$$

Except for the case when $a$ is a complete intersection, in which case the Koszul complex provides a resolution of $\mathcal{O} /(a)$, the inclusion (2.6) is strict, see [28] and [19]. Nevertheless, the singularities of $R^{a}$ reflect the characteristic varieties associated to the ideal, see [19] and [10], which are closely related to the integral closure of powers of $(a)$, and therefore $R^{a}$ is well suited for the Briançon-Skoda theorem. 
A slight modification of the Koszul complex, derived from the socalled Eagon-Northcott complex, with associated ideal sheaf $\mathcal{J}=(a)^{\ell}$, was introduced in [4]. The associated form $u^{a, \ell}$ is a sum of terms like

$$
\frac{\bar{a}_{I_{1}} \cdots \bar{a}_{I_{\ell}} \bar{\partial} \bar{a}_{I_{\ell+1} \wedge \ldots \wedge} \bar{\partial}_{I_{I_{k+\ell-1}}}}{|a|^{2(k+\ell-1)}}, \quad k \leq m
$$

see the proof of Theorem 1.1 in [4] for a precise description of $u^{a, \ell}$ and the corresponding residue current $R^{a, \ell}$. It turns out that $\phi$ annihilates $R^{a, \ell}$ if (1.1) holds, and thus $\phi \in(a)^{\ell}$, so the classical Briançon-Skoda theorem follows. The most expedient way to prove this annihilation is to use a resolution of singularities where $a$ is principal. However, it is not really necessary to define the current $R^{a, \ell}$ in itself; it is actually enough to make sure that $\phi R^{a, \ell, \delta} \rightarrow 0$ when $\delta \rightarrow 0$, and this can be proved essentially by integration by part in an ingenious way, thus providing a proof of the Briançon-Skoda theorem by completely elementary means, see [27].

In [8] was introduced the sheaf of pseudomeromorphic currents $\mathcal{P} \mathcal{M}$. For the definition, see [8]. It is closed under $\bar{\partial}$ and multiplication with smooth forms. In particular, the currents $U$ and $R$ are pseudomeromorphic. The following fact (Corollary 2.4 in [8]) will be used repeatedly.

Proposition 2.1. If $T \in \mathcal{P} \mathcal{M}$ has bidegree $(r, k)$ and the support of $T$ is contained in a variety of codimension strictly larger than $k$, then $T=0$.

In particular, this means that if $Z$ (the variety where (2.1) is not pointwise exact) has codimension $p$ then $R=R_{p}+R_{p+1}+\cdots$.

As mentioned in the introduction, we need to form products of currents associated to complexes. Assume therefore that $\left(\mathcal{O}\left(E_{\bullet}^{g}\right), g_{\bullet}\right)$ and $\left(\mathcal{O}\left(E_{\bullet}^{h}\right), h_{\bullet}\right)$ are two complexes as above and $\mathcal{I}$ and $\mathcal{J}$ are the corresponding ideal sheaves. We can define a complex (2.1) with

$$
E_{k}=\bigoplus_{i+j=k} E_{i}^{g} \otimes E_{j}^{h}
$$

and $f=g+h$, or more formally, $f=g \otimes I_{E^{h}}+I_{E^{g}} \otimes h$, such that

$$
f(\xi \otimes \eta)=g \xi \otimes \eta+(-1)^{\operatorname{deg} \xi} \xi \otimes h \eta .
$$

Notice that $E_{0}=E_{0}^{g} \otimes E_{0}^{h}=\mathbb{C}$ and that $f_{1} \mathcal{O}\left(E_{1}\right)=\mathcal{I}+\mathcal{J}$. One can extend (2.8) to form-valued or current-valued sections $\xi$ and $\eta$ and $\operatorname{deg} \xi$ then means total degree. It is natural to write $\xi \wedge \eta$ rather than $\xi \otimes \eta$, and we define $\eta \wedge \xi$ as $(-1)^{\operatorname{deg} \xi \operatorname{deg} \eta} \xi \wedge \eta$. Notice that

$$
\nabla_{f}(\xi \otimes \eta)=\nabla_{g} \xi \otimes \eta+(-1)^{\operatorname{deg} \xi} \xi \otimes \nabla_{h} \eta .
$$

Let $u^{g}$ and $u^{h}$ be the corresponding $E^{g}$-valued and $E^{h}$-valued forms, cf. (2.2). Then $u=u^{h} \wedge u^{g}$ is an $E$-valued form outside $Z^{g} \cup Z^{h}$. Following 
the proof of Proposition 2.1 in [8] we can define $E$-valued pseudomeromorphic currents

$$
R^{h} \wedge R^{g}=\lim _{\delta \rightarrow 0} R^{h, \delta} \wedge R^{g}, \quad U^{h} \wedge R^{g}=\lim _{\delta \rightarrow 0} U^{h, \delta} \wedge R^{g},
$$

where $U^{h, \delta}=\chi_{\delta} u^{h}$ and $R^{h, \delta}=1-\chi_{\delta}+\bar{\partial} \chi_{\delta} \wedge u^{h}$, and $\chi_{\delta}=\chi\left(|H|^{2} / \delta^{2}\right)$ as before. The "product" $R^{h} \wedge R^{g}$ so defined is not equal to $R^{g} \wedge R^{h}$ in general. It is also understood here that $H$ only vanishes where it has to, i.e., on the set where the complex $\left(E_{\bullet}^{h}, h_{\bullet}\right)$ is not pointwise exact. If we use an $H$ that vanishes on a larger set, the result will be affected. It is worth to point out that a certain component $R_{k}^{h} \wedge R^{g}$ may be nonzero even if $R_{k}^{h}$ itself vanishes.

Proposition 2.2. With the notation above we have that

$$
\nabla_{f}\left(U^{g}+U^{h} \wedge R^{g}\right)=1-R^{h} \wedge R^{g} .
$$

Moreover, $\phi R^{h} \wedge R^{g}=0$ implies that $\phi \in \mathcal{I}+\mathcal{J}$.

Proof. Recall that $\nabla_{h} U^{h, \delta}=1-R^{h, \delta}, \nabla_{g} U^{g}=1-R^{g}$ and $\nabla_{g} R^{g}=0$. Therefore,

$$
\nabla_{f}\left(U^{g}+U^{h, \delta} \wedge R^{g}\right)=1-R^{g}+\left(1-R^{h, \delta}\right) \wedge R^{g}=1-R^{h, \delta} \wedge R^{g} .
$$

Taking limits, we get (2.10). The second statement now follows in the same way as (2.4) above.

\section{The Residue CURRENT Associated to the VARIETy $Z$}

Consider a subvariety $Z$ of a neighborhood of the origin in $\mathbb{C}^{n}$ with radical ideal sheaf $\mathcal{I}$ and let (1.4) be a resolution of $\mathcal{O} / \mathcal{I}$. Let $R^{Z}$ be the associated residue current obtained as in the previous section. We then know that $R^{Z}$ has support on $Z$ and that ann $R^{Z}=\mathcal{I}$. Outside the set $Z_{k}$, cf., Section 1, the mapping $f_{k}$ has constant rank, and hence $\sigma_{k}$ is smooth there. Outside $Z_{k}$ we therefore have that

$$
R_{k+1}^{Z}=\alpha_{k+1} R_{k}^{Z}
$$

where $\alpha_{k+1}=\bar{\partial} \sigma_{k+1}$ is a $\operatorname{smooth} \operatorname{Hom}\left(E_{k}, E_{k+1}\right)$-valued $(0,1)$-form, cf., $(2.2)$.

Locally on $Z_{\text {reg }}$, the current $R^{Z}$ is essentially the integration current $[Z]$. We have the following more precise statement that gives a Dolbeault-Lelong-type representation, in the sense of [12], of the current $R^{Z}$. Let $\chi$ be a smooth regularization of the characteristic function of $[1, \infty)$ and $p=\operatorname{codim} Z$ as before.

Proposition 3.1. For each given $x \in Z_{\text {reg, }}$, there is a hypersurface $\{h=0\}$ in $Z$, avoiding $x$ but containing $Z_{\text {sing }}$ and intersecting $Z$ properly, and $E_{k}$-valued $(n-p, k-p)$-forms $\beta_{k}$, smooth outside $\{h=0\}$, such that

$$
R_{k}^{Z} \cdot(d z \wedge \xi)=\lim _{\epsilon \rightarrow 0} \int_{Z} \chi(|h| / \epsilon) \beta_{k} \wedge \xi, \quad \xi \in \mathcal{D}_{0, n-k}(X),
$$


for $p \leq k \leq n$. Moreover, in a suitable resolution $\pi: \tilde{Z} \rightarrow Z$ the forms $\beta_{k}$ locally have the form $\alpha_{k} / m_{k}$, where $\alpha_{k}$ are smooth and $m_{k}$ are monomials.

Here, $d z=d z_{1} \wedge \cdots \wedge d z_{n}$.

Proof. Following Section 5 in [6] (the proof of Proposition 2.2) one can find, for each given $x \in Z_{\text {reg }}$, a holomorphic function $h$ such that $h(x) \neq 0$ and $h$ does not vanish identically on any component of $Z_{\text {reg }}$. Moreover, for $k \geq p$,

$$
\left.R_{k}^{Z}=\gamma_{k}\right\lrcorner[Z]
$$

where $\gamma_{k}$ is an $E_{k}$-valued and $(0, k-p)$-form-valued $(p, 0)$-vector field that is smooth outside $\{h=0\}$. Let $\xi$ be a test form of bidegree $(0, n-$ $k$ ). The current $R^{Z}$ has the so-called standard extension property, SEP, see [8] Section 5, which means that

$$
\left.\left.R_{k}^{Z} \cdot(\xi \wedge d z)=\lim _{\epsilon \rightarrow 0} \int \chi(|h| / \epsilon) \gamma_{k}\right\lrcorner[Z] \wedge \xi \wedge d z= \pm \lim _{\epsilon \rightarrow 0} \int_{Z} \chi(|h| / \epsilon) \xi \wedge \gamma_{k}\right\lrcorner d z .
$$

Thus we can take $\left.\beta_{k}= \pm \gamma_{k}\right\lrcorner d z$.

More precisely, according to the last paragraph of Section 5 in [6], $\gamma_{p}$ is a meromorphic $(p, 0)$-field (with poles where $h=0$ ) composed by the orthogonal projection of $E_{p}$ onto the orthogonal complement in $E_{p}$ of the pointwise image of $f_{p+1}$. This projection is given by

$$
I_{E_{p}}-f_{p+1} \sigma_{p+1} \text {. }
$$

Furthermore, cf., (3.1),

$$
\gamma_{k}=\left(\bar{\partial} \sigma_{k}\right) \cdots\left(\bar{\partial} \sigma_{p+1}\right) \gamma_{p}
$$

for $k>p$. Now choose a resolution of singularities $\tilde{Z} \rightarrow Z$ such that for each $k$ the the determinant ideal of $f_{k}$ is principal. On $\tilde{Z}$, then each $\sigma_{k}$ (locally) is a smooth form over a monimial, see Section 2 in [7], and thus $\left.\beta_{k}=\gamma_{k}\right\lrcorner d z$ has this form as well.

We can choose the resolution of singularities $\tilde{Z} \rightarrow Z$ so that also $\tilde{h}=$ $\pi^{*} h$ is a monomial. By a partition of unity it follows that $R_{k}^{Z} \cdot(d z \wedge \xi)$ is a finite sum of terms like

$$
\lim _{\epsilon \rightarrow 0} \int_{s} \chi(|\tilde{h}| / \epsilon) \frac{d s_{1} \wedge \ldots \wedge d s_{\nu}}{s_{1}^{\alpha_{1}+1} \ldots s_{\nu}^{\alpha_{\nu}+1}} \wedge \tilde{\xi} \wedge \psi
$$

where $s_{1}, \ldots, s_{n-p}$ are local holomorphic coordinates and $\nu \leq n-p, \tilde{\xi}=$ $\pi^{*} \xi$, and $\psi$ is a smooth form with compact support. It is easily checked that this limit is the tensor product of the one-variable principal value currents $d s_{i} / s_{i}^{\alpha_{i}+1}, 1 \leq j \leq \nu$, acting on $\tilde{\xi} \wedge \psi$. Therefore (3.2) is equal to (a constant times)

$$
\int \frac{d s_{1} \wedge \ldots \wedge d s_{\nu}}{s_{1} \cdots s_{\nu}} \wedge \partial_{s}^{\alpha}(\tilde{\xi} \wedge \psi),
$$

if $\partial_{s}^{\alpha}=\partial_{s_{1}}^{\alpha_{1}} \cdots \partial_{s_{\nu}}^{\alpha_{\nu}}$. 


\section{Proof of Theorem 1.1}

To prove Theorem 1.1 we are going to apply the idea in Example 2 but performed on $Z$. To this end we assume that $Z$ is embedded in $\mathbb{C}^{n}$ and we let $R^{Z}$ be the current introduced in the previous section. Let $\mathfrak{a}=(a)$ be the ideal in $\mathcal{O}_{Z}$ and suppose for the moment that $a$ also denotes representatives in $\mathcal{O}$ of the generators. If $R^{a, \ell}=\lim _{\delta \rightarrow 0} R^{a, \ell, \delta}$ denotes the current from Example 2 we can form, cf., the end of Section 2, the product

$$
R^{a, \ell} \wedge R^{Z}=\lim _{\delta \rightarrow 0} R^{a, \ell, \delta} \wedge R^{Z} .
$$

Since $R^{Z}$ annihilates $\mathcal{I}$ it follows that $R^{a, \ell} \wedge R^{Z}$ only depends on $\mathfrak{a} \subset \mathcal{O}_{Z}$. For the same reason, $\phi R^{a, \ell} \wedge R^{Z}$ is well-defined for $\phi \in \mathcal{O}_{Z}$. We know from Proposition 2.2 that $\phi$ belongs to $\mathfrak{a}$ if it annihilates this current, and thus Theorem 1.1 follows from the following proposition.

Proposition 4.1. If the hypotheses of Theorem 1.1 are fulfilled i.e., either (1.6), or (1.8) together with the geometric conditions (1.7), then $\phi R^{a, \ell} \wedge R^{Z}=0$.

Remark 2. It is natural to try to use the Lelong current $[Z]$ rather than

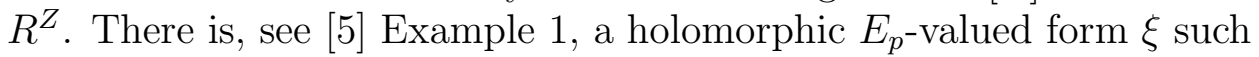
that $[Z]=\xi \cdot R_{p}^{Z}$. Thus the hypotheses in Theorem 1.1 imply that $\phi R^{a} \wedge[Z]=0$. However, this in turn does not imply that $\phi$ is in $(a)$. In fact, if $m=1$ so that $a$ is just one function, then

$$
0=\phi R^{a} \wedge[Z]=\phi \bar{\partial} \frac{1}{a} \wedge[Z]
$$

and this means that $\phi / a$ is in $\omega_{Z}^{0}$ introduced by Barlet, see, e.g., [16], and this class is wider than $\mathcal{O}_{Z}$ in general.

Proof of Proposition 4.1. We first assume that (1.7) and (1.8) hold. Considering $\phi R^{a, \ell}$ as an intrinsic current on the submanifold $Z_{\text {reg }}$ (cf. the beginning of this section) it follows from the residue proof of the Briançon-Skoda theorem in the regular case that $\phi R^{a, \ell}$ must vanish on $Z_{\text {reg }}$ since (1.8) holds. Thus, $\phi R^{a, \ell} \wedge[Z]$ vanishes on $Z_{\text {reg }}$ and so, in view of Proposition 3.1, it follows that the support of $\phi R^{a, \ell} \wedge R^{Z}$ is contained in $Z_{\text {sing. }}$. On the other hand it is readily verified that $R^{a, \ell} \wedge R^{Z}$ must vanish if $a$ is nonvanishing. Thus the support of $\phi R^{a, \ell} \wedge R^{Z}$ is contained in $Z_{\text {sing }} \cap Z^{a}$.

The current $R^{a, \ell}$ has (maximal) bidegree $(0, m)$ and hence $R^{a, \ell} \wedge R_{p}^{Z}$ has (maximal) bidegree $(0, m+p)$. Since it has support on $Z_{\text {sing }} \cap Z^{a}$ that has codimension $\geq p+m+1$ by (1.7), it follows that $\phi R^{a, \ell} \wedge R_{p}^{Z}=0$. Outside $Z_{p+1}$ we have that $R_{p+1}^{Z}=\alpha_{p+1} R_{p}^{Z}$ for a smooth form $\alpha_{p+1}$, and hence

$$
\phi R^{a, \ell} \wedge R_{p+1}^{Z}=\phi R^{a, \ell} \wedge \alpha_{p+1} R_{p}^{Z}=\alpha_{p+1} \phi R^{a, \ell} \wedge R_{p}^{Z}=0
$$


there. Thus $\phi R^{a, \ell} \wedge R_{p+1}^{Z}$ has support on $Z_{p+1} \cap Z^{a}$, and again for degree reasons we find that $\phi R^{a, \ell} \wedge R_{p+1}^{Z}=0$. Continuing in this way we can conclude that $\phi R^{a, \ell} \wedge R^{Z}=0$.

We now assume that (1.6) holds. We have to prove that $R^{Z} .(d z \wedge \xi) \rightarrow$ 0 when $\delta \rightarrow 0$, for

$$
\xi=\phi R^{a, \ell, \delta} \wedge \eta
$$

with test forms $\eta$ of bidegree $(0, *)$. In view of the comments after the proof of Proposition 3.1 it is enough to prove that each term (3.3) tends to zero if (1.6) holds and $\mu$ is large enough (independently of $(a)$ and $\ell)$. For this particular term we will see that we need $\mu \geq \mu_{0}$, where

$$
\mu_{0}=|\alpha|+2 \min (m, n-p) .
$$

For simplicity we omit all snakes from now on and write $\phi$ rather than $\tilde{\phi}$ etc. Moreover, we assume that $\ell=1$, the general case follows completely analogously. Since $\tilde{Z}$ is smooth, by the usual Briançon-Skoda theorem we have that

$$
\phi \in(a)^{|\alpha|+\min (m, n-p)+1} .
$$

Notice that

$$
R_{k}^{a, \delta}=\chi^{\prime}\left(|a|^{2} / \delta^{2}\right) \wedge \frac{\bar{\partial}|a|^{2}}{\delta^{2}} \wedge u_{k}^{a}, \quad k>0,
$$

and thus $R_{k}^{a, \delta}$ is a sum of terms like

$$
\chi^{\prime} \frac{\bar{\partial} \bar{a}_{I_{1}} \wedge \ldots \wedge \bar{\partial} \bar{a}_{I_{k}}}{\delta^{2}|a|^{2 k}} \bar{a} a \wedge \omega
$$

for $|I|=k$, where in what follows $a^{r}$ denotes a product of $r$ factors $a_{i}$, and similarly with $\bar{a}^{r}$, and $\omega$ denotes a smooth form. For degree reasons $k \leq \nu=\min (m, n-p)$. In view of (4.3) therefore $\phi R_{k}^{a, \delta}$ is a sum of terms like

$$
\chi^{\prime} \frac{\bar{\partial} \bar{a}_{I_{1}} \wedge \ldots \wedge \bar{\partial} \bar{a}_{I_{\nu}}}{\delta^{2}|a|^{2 \nu}} \bar{a} a^{2+\nu+|\alpha|} \wedge \omega
$$

plus lower order terms. A straight forward computation yields that $\partial_{s}^{\alpha}\left(\phi R_{k}^{a, \delta}\right)$ is a finite sum of terms like

$$
\chi^{(r+1)} \frac{\bar{\partial} \bar{a}_{I_{1}} \wedge \ldots \wedge \bar{\partial} \bar{a}_{I_{\nu}}}{\delta^{2(r+1)}|a|^{2(\nu+|\gamma|-r)}} \bar{a}^{1+|\gamma|} a^{2+\nu+|\gamma|} \wedge \omega
$$

where $\gamma \leq \alpha$ and $r \leq|\gamma|$, plus lower order terms.

We thus have to see that each

$$
\int_{s} \frac{d s_{1} \wedge \ldots \wedge d s_{\nu}}{s_{1} \cdots s_{\nu}} \chi^{(r+1)} \frac{\bar{\partial} \bar{a}_{I_{1}} \wedge \ldots \wedge \bar{\partial} \bar{a}_{I_{\nu}}}{\delta^{2(r+1)}|a|^{2(\nu+|\gamma|-r)}} \bar{a}^{1+|\gamma|} a^{2+\nu+|\gamma|} \wedge \omega
$$


tends to 0 when $\delta \rightarrow 0$. After a suitable further resolution we may assume that locally $a=a_{0} a^{\prime}$ where $a_{0}$ is holomorphic and $a^{\prime}$ is a nonvanishing tuple. Then

$$
\bar{\partial} \bar{a}_{I_{1}} \wedge \ldots \wedge \bar{\partial} \bar{a}_{I_{\nu}}=\bar{a}_{0}^{\nu-1} \wedge \omega
$$

Also notice that the expression

$$
\frac{d s_{1} \wedge \ldots \wedge d s_{\nu}}{s_{1} \cdots s_{\nu}}
$$

becomes a sum of similar expressions in this new resolution. Altogether we end up with a finite sum of terms like

$$
\int_{s} \frac{d s_{1} \wedge \ldots \wedge d s_{\nu}}{s_{1} \cdots s_{\nu}} \chi^{(r+1)}\left(|a|^{2} / \delta^{2}\right) \wedge \mathcal{O}(1)
$$

and each such integral tends to zero by dominated convergence.

The term corresponding to $R_{0}^{a, \delta}=1-\chi\left(|a|^{2} / \delta^{2}\right)$ is handled in a similar but easier way.

\section{INTEGRAL REPRESENTATION OF THE MEMBERSHIP}

Finally we describe how one can obtain an explicit integral representation of the membership provided that the residue is annihilated. The starting point is the formalism in [2] to generate integral representations for holomorphic functions. Let $\delta_{\eta}$ denote interior multiplication with the vector field

$$
2 \pi i \sum_{1}^{n}\left(\zeta_{j}-z_{j}\right) \frac{\partial}{\partial \zeta_{j}}
$$

and let $\nabla_{\eta}=\delta_{\eta}-\bar{\partial}$. A smooth form $g=g_{0}+g_{1}+\cdots+g_{n}$, where $g_{k}$ has bidegree $(k, k)$, is called a weight (with respect to $z$ ) if $\nabla_{\eta} g=0$ and $g_{0}(z, z)=1$. Notice that the product of two weights is again a weight.

Example 3. Let $\chi$ be a cutoff function that is identically 1 in a neighborhood of the closed unit ball, and let

$$
s=\frac{1}{2 \pi i} \frac{\partial|\zeta|^{2}}{|\zeta|^{2}-\bar{\zeta} \cdot z} .
$$

Then $\nabla_{\eta} s=1-\bar{\partial} s$ and therefore

$$
g=\chi-\bar{\partial} \chi \wedge\left[s+s \wedge \bar{\partial} s+\cdots+s \wedge(\bar{\partial} s)^{n-1}\right]
$$

is a weight with respect to $z$ for each $z$ in the ball, with compact support, and it depends holomorphically on $z$.

If $g$ is a weight with compact support and $z$ is holomorphic on the support, then

$$
\phi(z)=\int g \phi=\int g_{n} \phi .
$$

Now consider a complex like (2.1) in Section 2, defined in a neighborhood of the closed ball, and let $U^{\delta}$ and $R^{\delta}$ be the associated $E$-valued 
forms. One can find, see [3] Proposition 5.3, holomorphic $E_{k}^{*}$-valued $(k, 0)$-forms $H_{k}^{0}$ and $\operatorname{Hom}\left(E_{k}, E_{1}\right)$-valued $(k-1,0)$-forms $H_{k}^{1}$ such that $\delta_{\eta} H_{k}^{0}=H_{k-1}^{0} f_{k}(\zeta)-f_{1}(z) H_{k}^{1}$ and $H_{j}^{j}=\operatorname{Id}_{E_{j}}$. Using that $\nabla_{f} U^{\delta}=1-R^{\delta}$ one verifies that

$$
f_{1}(z) H U^{\delta}+H R^{\delta}=1-\nabla_{\eta}\left(\sum H_{k}^{0} U_{k}^{\delta}\right)
$$

where

$$
H U^{\delta}=\sum H_{k}^{1} U_{k}^{\delta}, \quad H R^{\delta}=\sum H_{k}^{0} R_{k}^{\delta} .
$$

It follows that $g^{\delta}:=f_{1}(z) H U^{\delta}+H R^{\delta}$ is a weight with respect to $z$. If $g$ is, e.g., the weight from Example 3 we thus get the representation

$$
\phi(z)=\int g^{\delta} \wedge g \phi=f_{1}(z) \int H U^{\delta} \wedge g \phi+\int H R^{\delta} \wedge g \phi .
$$

Taking limits we obtain the interpolation-division formula

$$
\phi(z)=f_{1}(z) \int H U \wedge g \phi+\int H R \wedge g \phi .
$$

To be precise, the integrals here are the action of currents on smooth forms. In particular, (5.1) implies that $\phi$ belongs to the ideal generated by $f_{1}$ if $\phi R=0$.

If we now choose as our complex the resolution of the sheaf $I=I_{Z}$, we get the formula

$$
\phi(z)=\int g \wedge H^{Z} R^{Z} \phi, \quad z \in Z,
$$

for $\phi \in \mathcal{O}_{Z}$. We then replace $g$ by the weight $g^{a, \ell, \delta} \wedge g$, where

$$
g^{a, \ell, \delta}=a(z)^{\ell} \cdot H^{a, \ell} U^{a, \ell, \delta}+H^{a, \ell} R^{a, \ell, \delta} ;
$$

here $a(z)^{\ell}$ denotes the first mapping in the complex associated with $(a)^{\ell}$, cf., Example 2, so that its entries are elements in the ideal $(a)^{\ell}$. We get

$$
\begin{aligned}
\phi(z)= & a(z)^{\ell} \cdot \int_{\zeta} H^{a} U^{a, \ell, \delta} \wedge H^{Z} R^{Z} \phi \wedge g \\
& +\int_{\zeta} H^{a} R^{a, \ell, \delta} \wedge H^{Z} R^{Z} \wedge g \phi .
\end{aligned}
$$

If the hypotheses in Theorem 1.1 are fulfilled, since $H^{Z}, H^{a}$ and $g$ are smooth, the second integral tends to zero when $\delta \rightarrow 0$, and the first integral on the right hand side converges to an $E_{1}^{a, \ell}$-valued holomorphic function. Thus we get the explicit representation

$$
\phi(z)=a(z)^{\ell} \cdot \int_{\zeta} H^{a} U^{a, \ell} \wedge H^{Z} R^{Z} \phi \wedge g
$$

of the membership. 


\section{REFERENCES}

[1] M. Andersson: Residue currents and ideals of holomorphic functions, Bull. Sci. Math., 128 (2004), 481-512.

[2] M. Andersson: Integral representation with weights I, Math. Ann., 326 (2003), 1-18.

[3] M. Andersson: Integral representation with weights. II. Division and interpolation, Math. Z., 254 (2006), 315-332.

[4] M. Andersson: Explicit versions of the Briancon-Skoda theorem with variations, Michigan Math. J., 54(2) (2006), 361-373.

[5] M. Andersson: Coleff-Herrera currents, duality, and Noetherian operators, Preprint Gothenburg (2009), available at arXiv:0902.3064.

[6] M. Andersson, H. SAmuelsson: Koppelman formulas and the $\bar{\partial}$ equation on an analytic space, Preprint Mittag-Leffler (2008), available at arXiv:0801.0710.

[7] M. Andersson, E. WulcAn: Residue currents with prescribed annihilator ideals, Ann. Sci. École Norm. Sup., 40 (2007), 985-1007.

[8] M. Andersson, E. Wulcan: Decomposition of residue currents, J. reine angew. Math., (to appear), available at arXiv:0710.2016.

[9] M. Andersson: A residue criterion for strong holomorphicity, Preprint Gothenburg 2007, available at arXiv:0711.2863.

[10] M. Andersson, E. GöTMARK: Explicit representation of membership of polynomial ideals, Preprint Mittag-Leffler (2008), available at arXiv:0806.2592.

[11] C. Berenstein, R. Gay, A. Vidras, A. Yger: Residue Currents and Bézout Identities, Birkhäuser (1993).

[12] J.-E. BJöRK: Residue currents and D-modules, The legacy of Niels Henrik Abel, 605-651, Springer, Berlin 2004.

[13] J. Briançon, H. Skoda: Sur la clôture intégrale d'un idéal de germes de fonctions holomorphes en un point de $\mathbb{C}^{n}$, C. R. Acad. Sci. Paris Sér. A 278 (1974), 949-951.

[14] N.R. Coleff, M.e. Herrera: Les courants résiduels associés à une forme méromorphe, Lect. Notes in Math. 633, Berlin-Heidelberg-New York (1978).

[15] D. EISENBUD: Commutative algebra. With a view toward algebraic geometry, Graduate Texts in Mathematics, 150. Springer-Verlag, New York, 1995.

[16] G. Henkin, M. Passare: Abelian differentials on singular varieties and variatiosn on a theorem of Lie-Griffiths, Invent. Math., 135 (1999), 297328.

[17] C. Huneke: Uniform bounds in Noetherian rings, Invent. Math., 107 (1992), 203-223.

[18] E. Hyry, O. Villamayor: A Briançon-Skoda Theorem for Isolated Singularities, J. Algebra, 204 (1998), 656-665.

[19] M. Jonsson, E. Wulcan: On Bochner-Martinelli residue currents and their annihilator ideals, arXiv:0811.0636 .

[20] M. Lejeune-Jalabert, B. Teissier, J.-J. Risler: Clôture intégrale des idéaux et équisingularité, arXiv:0803.2369.

[21] J. Lipman, A. Sathaye: Jacobian ideals and a theorem of Briançon-Skoda, Michigan Math. J., 28 (1981), no. 2, 199-222.

[22] J. Lipman, B. Teissier: Pseudorational local rings and a theorem of Briançon-Skoda about integral closures of ideals, Michigan Math. J., 28 (1981), 97-116.

[23] M. Passare, A. Tsikh, A. YGer: Residue currents of the BochnerMartinelli type, Publ. Mat., 44 (2000), 85-117. 
[24] H. SAmuelsson: Regularizations of products of residue and principal value currents, J. Funct. Anal., 239 (2006), 566-593.

[25] G. ScheJA: Riemannsche Hebbarkeitssätze für Cohomologieklassen, Math. Ann., 144 (1961), 345-360.

[26] H. SKodA: Application des techniques $L^{2}$ à la théorie des idéaux d'une algèbre de fonctions holomorphes avec poids, Ann. Sci. École Norm. Sup., 5 (1972), 545-579.

[27] J. Sznajdman: An elementary proof of the Briançon-Skoda theorem, Preprint Gothenburg 2008, Available at arXiv:0807.0142.

[28] E. Wulcan: Residue currents of monomial ideals, Indiana Univ. Math. J., 56 (2007), 365-388.

Department of Mathematics, Chalmers University of Technology And University of Gothenburg, S-412 96 GÖTEBORG, SWEDEN

E-mail address: matsa@math.chalmers.se, hasam@math.chalmers.se, sznajdma@math.chalmers.se 